\title{
An Ultrawideband (UWB) Switched-Antenna-Array Radar Imaging System
}

\author{
Gregory L. Charvat, \\ Leo C. Kempel, \\ and Edward. J. Rothwell \\ Dept. of Electrical \\ and Computer Engineering \\ Michigan State University \\ 2120 Engineering Building \\ East Lansing, MI, 48824 \\ E-mail: gregory.charvat@1l.mit.edu, \\ kempel@egr.msu.edu, \\ and rothwell@egr.msu.edu
}

\author{
Christopher M. Coleman \\ Integrity Applications Incorporated \\ 5180 Parkstone Drive, Suite 260 \\ Chantilly, VA 20151 \\ E-mail: ccoleman@integrity-apps.com
}

\author{
Eric L. Mokole \\ Naval Research Laboratory \\ Attn: Code 5340 (E. Mokole) \\ 4555 Overlook Avenue S.W. \\ Washington, DC 20375 \\ E-mail: eric.mokole@nrl.navy.mil
}

\begin{abstract}
A low-cost ultrawideband (UWB), 1.926-4.069 GHz, phased array radar system is developed that requires only one exciter and digital receiver that is time-division-multiplexed (TDM) across 8 receive elements and 13 transmit elements, synthesizing a fully populated $2.24 \mathrm{~m}$ long $(\lambda / 2$ element-toelement spacing) linear phased array. A $2.24 \mathrm{~m}$ linear phased array with a $3 \mathrm{GHz}$ center frequency would require 44 antenna elements but this system requires only 21 elements and time to acquire bi-static pulses across a subset of element combinations. This radar system beamforms in the near field, where the target scene of interest is located $3-70 \mathrm{~m}$ down range. It utilizes digital beamforming, computed using the range migration synthetic aperture radar (SAR) algorithm. The phased array antenna is fed by transmit and receive fan-out switch matrices that are connected to a UWB LFM pulse compressed radar operating in stretch mode. The peak transmit power is $1 \mathrm{~mW}$ and the transmitted LFM pulses are long in time duration $(2.5-10 \mathrm{~ms})$, requiring the radar to transmit and receive simultaneously. It will be shown through simulation and measurement that the bi-static antenna pairs are nearly equivalent to 44 elements spaced $\lambda / 2$ across a linear array. This result is due to the fact that the phase center position errors relative to a uniform $\lambda / 2$ element spacing are negligible. This radar is capable of imaging free-space target scenes made up of objects as small as $15.24 \mathrm{~cm}$ tall rods and 3.2 cm tall metal nails at a $0.5 \mathrm{~Hz}$ rate. Applications for this radar system include short-range near-real-time imaging of unknown targets through a lossy dielectric slab and radar cross section (RCS) measurements.
\end{abstract}

\section{INTRODUCTION}

It was shown in [1] that SAR imaging through solid concrete is feasible at stand-off ranges when a modified frequency modulated continuous wave (FMCW) radar architecture is used as a linear rail synthetic aperture radar (SAR). Targets as small as soda cans could be detected and localized through a $10 \mathrm{~cm}$ solid concrete wall at a stand-off range of 9.1 m. Modeling agreed with measured results, showing that the attenuation through the concrete slab was the greatest challenge and the wall dielectric properties need not be a part of the imaging algorithm when operating at stand-off ranges.
This FMCW radar architecture facilitated stand-off ranges by providing a relatively high average transmit power of $2 \mathrm{~mW}$, but low peak-power of $10 \mathrm{~mW}$, by use of long-duration (2.5$10 \mathrm{~ms}$ ) linear frequency modulated (LFM) transmit pulses and a short-duration range gate implemented through use of highcircuit-Q analog IF filters. This rail SAR proved to be effective at imaging through a concrete slab, unfortunately, it required approximately 20 minutes to acquire data. This duration of time is too slow for practical through-slab applications. In this paper a high-speed switched-antenna array implementation of this through-slab SAR will be shown, where the time to acquire a full through-slab data set is reduced from 20 minutes to 1.9 seconds.

Similar arrays used for short-range free-space radar imaging include [2]-[4]. Previous switched antenna array work used for through-slab radar imaging include [5]-[14]. The array shown in this paper is different than previous work because it facilitates $\lambda / 2$ element spacing and it is interfaced to the range-gated FMCW radar system from [1], enabling throughslab imaging at a stand-off range of $6 \mathrm{~m}$ or greater.

A conventional $2.24 \mathrm{~m}$ long array with $\lambda / 2$ spacing at 3 $\mathrm{GHz}$ using this FMCW radar solution would require 44 bistatic pairs of transmit and receive elements for a total of 88 elements spanning the length of the array. It will be shown that a $2.24 \mathrm{~m}$ long 21 element switched array provides 44 effective mono-static elements with a $\lambda / 2$ spacing, providing the same capability with less than a quarter as many elements. Switched array is achieved by time division multiplexing (TDM) the transmitter and receiver ports of the FMCW radar across a subset of the antenna element combinations. This data is fed to a SAR imaging algorithm that produces one radar image every (approximately) 1.9 seconds.

SAR imaging using a switched antenna array is discussed in Section II. The hardware implementation is presented in Section III. Calibration is discussed in Section IV. In Section $\mathrm{V}$, it will be shown that this phased array radar system is 
capable of imaging a point target with comparable range and cross-range sidelobes to that of a rail SAR. In addition to this, sensitivity in the near field is demonstrated by imaging groups of low RCS targets in free space using only $1 \mathrm{~mW}$ of peak transmit power. Summary and future work are discussed in Section VI.

\section{SWITCHED ANTENNA PHASED ARRAY}

The FMCW radar from [1] requires separate transmit and receive antennas, therefore, a $2.24 \mathrm{~m}$ long array providing $\lambda / 2$ element spacing at $3 \mathrm{GHz}$ would require a total of 88 antenna elements consisting of 44 transmit and 44 receive elements. This large number of elements significantly increases the cost and complexity of the system. Fortunately the practical application does not require instantaneous beamforming, therefore, the phased array is not required to provide a range profile simultaneously at every $\lambda / 2$ position. From this, a more efficient use of transmit and receive elements can be acheived by incorporating switched antenna array techniques resulting in $\lambda / 2$ spacing across the length of $2.24 \mathrm{~m}$ using only 8 receive elements and 13 transmit elements.

The transmit and receive ports of the FMCW radar from [1] are fed to two fan-out switch matrices that rout transmit signals to transmit elements and receive signals to receive elements (Fig. 1). The transmit fan-out switch matrix has approximately $10 \mathrm{~dB}$ of insertion loss, resulting in a $1 \mathrm{~mW}$ peak power at the transmit antenna elements. The receive fan-out switch matrix also has insertion loss, therefore, in order to preserve noise figure each receive element has an LNA connected to it.

The transmit and receive elements are physically separated into two sub-arrays, where the transmit sub-array includes elements ANT9-21 and the receive sub-array includes elements ANT1-8. Each element is a linear tapered slot antenna (LTSA) [15]-[21] etched on to FR-4 substrate according to the layout in Fig. 2.

The physical location of every element on the array is shown in Fig. 3. Each element is represented by the larger circles and labeled accordingly. The receive elements are on the top row and the transmit elements are on the bottom row.

At any given time the transmitter and receiver ports are routed to only one antenna respectively through the switch matrices. This provides bi-static radar transmitter and receiver element combinations. Only 44 combinations are used to synthesize a $\lambda / 2$ aperture, where, each of the bi-static combinations are shown in Fig. 3 by a straight lines between the transmitter and receiver elements. The bi-static combination of each pair of transmit and receive elements provides an effective mono-static element in between. The middle row of small circles in Fig. 3 indicates where the effective monostatic elements are located. The SAR imaging algorithm used is the range migration algorithm from [22]. This algorithm assumes that range profiles are acquired using these effective mono-static antenna elements forming a stripmap SAR data set identical to that from [1]. The imaging algorithm also assumes that the spacing between these effective mono-static elements is a uniform $5.08 \mathrm{~cm}$ which is approximately $\lambda / 2$ at

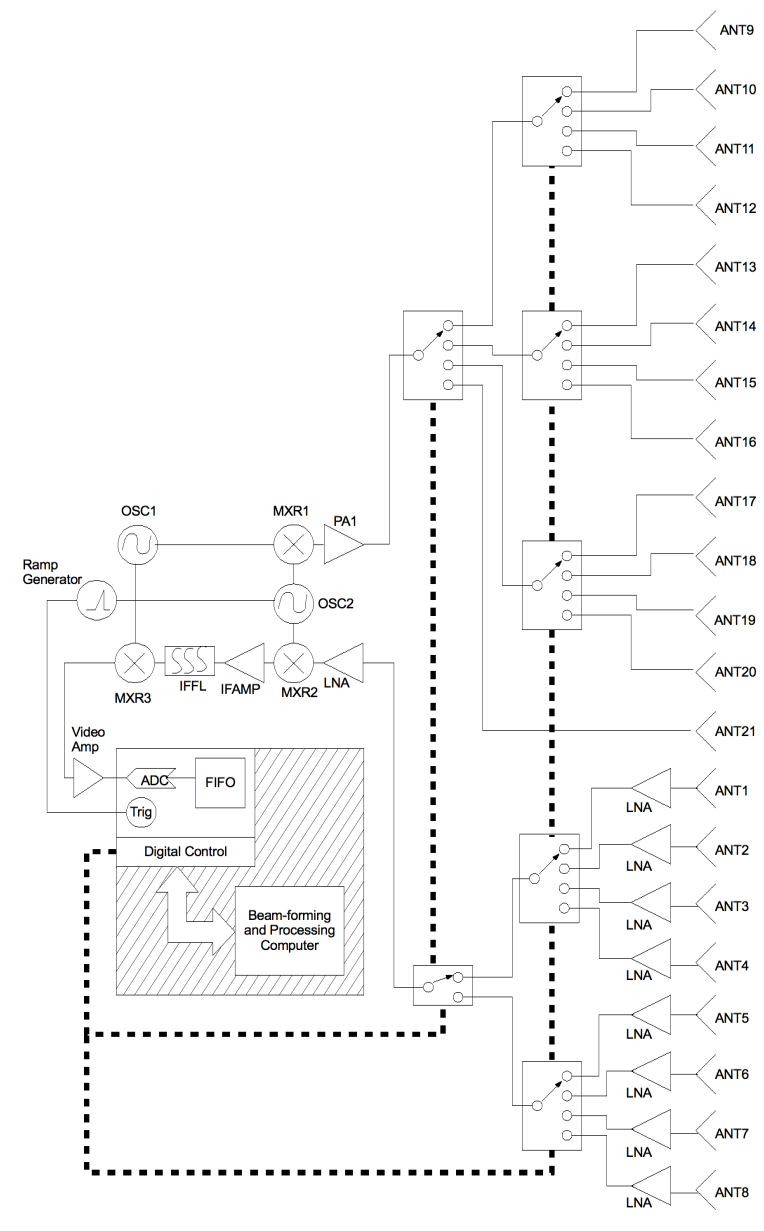

Fig. 1. Block diagram.

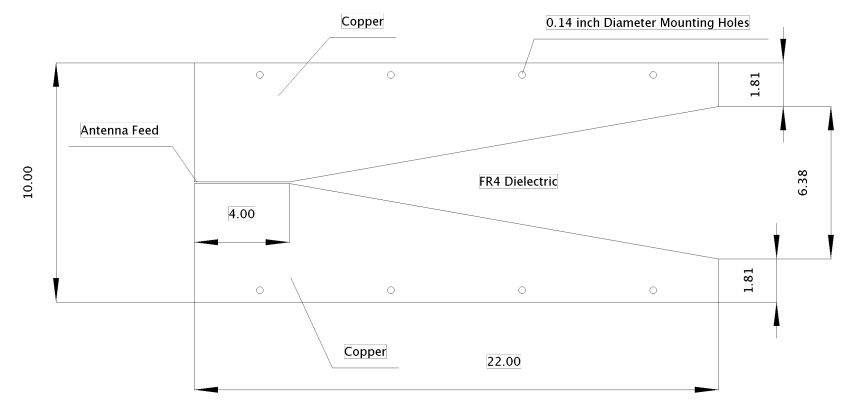

Fig. 2. Layout of the LTSA element used for both transmit and receive (units in inches).

the radar center frequency of $3 \mathrm{GHz}$. These assumptions are an approximation to the actual effective mono-static element position.

According to bi-static radar theory, given a separate transmitter and receiver the location of the effective mono-static element lies along the angle bisector of the triangle that is made up of the transmitter, receiver, and target [23]. For this 


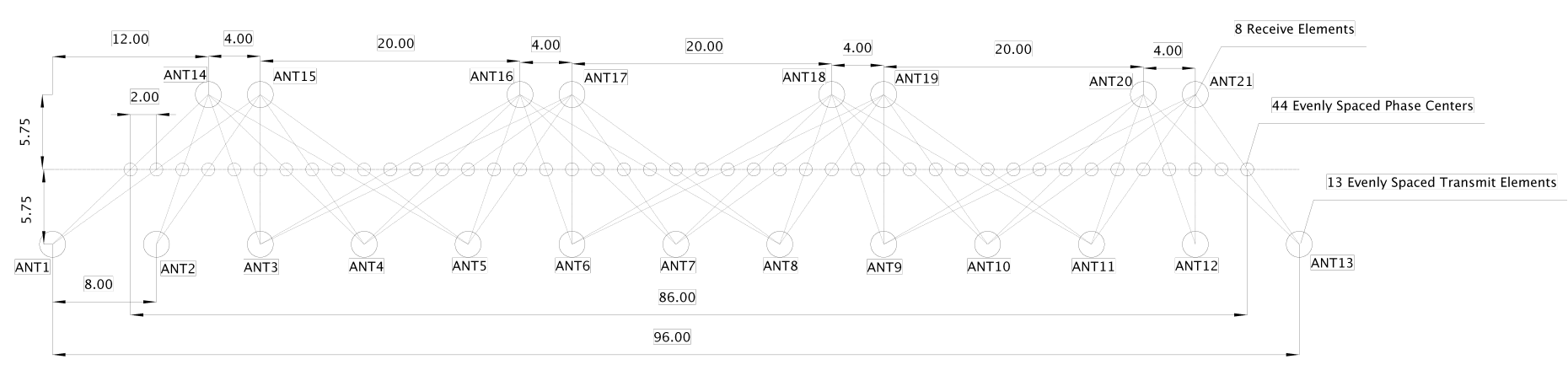

Fig. 3. The high speed SAR imaging array physical layout (units in inches), antenna combinations and phase center locations.

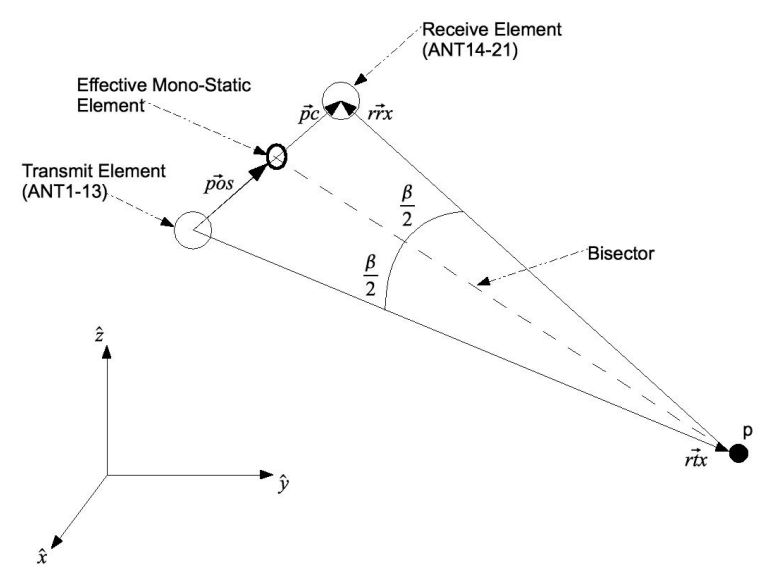

Fig. 4. Location of the effective mono-static element.

radar system, each bi-static antenna combination makes up a triangle with a point target somewhere down range in the scene represented by point $p$ in Fig. 4. Three points make up each triangle; the transmitter element, receive element, and $p$. The effective mono-static element due to the bi-static combination of the transmitter and receiver elements is located along the vector $\overrightarrow{p c}$. The location of the effective mono-static element along $\overrightarrow{p c}$ is the position vector $p \overrightarrow{o s}$ which depends on the length of $r \vec{r} x$ and $r \vec{t} x$ in the direction of $\overrightarrow{p c}$ as determined by the angle bisector theorem [24],

$$
p \vec{o} s=\frac{\overrightarrow{p c}}{\left(\frac{|\vec{r} x|}{|\vec{r} x|}+1\right)} .
$$

Equation 1 shows that the effective mono-static element spacing for this array is not precisely uniform given the physical layout of the bi-static antenna array and the shortrange geometry of the target scene that this radar system is designed to image. Using the actual layout of the array this offset error was calculated for a point target located at $p=(457.2,121.92,14.61) \mathrm{cm}$, where the origin of the coordinate system is located at ANT1 in Fig. 3 (the x-axis is down range away from the array, the y-axis is the length of the array, and the z-axis is the height of the array). The results are shown in Fig. 5, where the maximum y-axis error is $3 \mathrm{~mm}$ and the maximum z-axis error is $1.5 \mathrm{~mm}$. These errors are negligible compared to the $100 \mathrm{~mm}$ wavelength of the 3 $\mathrm{GHz}$ center frequency.

To assess the effect of these errors two SAR images of a point target at $p=(484.23,113.3,14.61) \mathrm{cm}$ were simulated. One data set was simulated assuming an error-free uniformly spaced linear array and the other was simulated using the actual array layout, where the effective mono-static elements are determined by Equation 1 .

A down-range cut of these images at the point target location shown in Fig. 6, shows that the errors are negligible between the switched array and a mono-static linear array with uniform element spacing.

A cross-range cut of these images at the point target location are shown in Fig. 7, showing that the peak magnitude of the point scatterer is $0.5 \mathrm{~dB}$ lower for the switched array than the uniformly spaced linear array. This simulation also shows that the first cross-range sidelobes of the switched array are approximately $2 \mathrm{~dB}$ lower than a uniformly spaced linear array.

This demonstrates that the switched array is a close approximation to a linear uniformly spaced array. Furthermore, it shows that the switched array has superior cross-range sidelobe performance compared to a uniformly spaced linear array. This is likely due to a slight randomization of phase centers. Measured results in Section V will show that measured azimuth and range sidelobes agree with simulation.

\section{HARDWARE IMPLEMENTATION}

Range-to-target information from a de-correlated FMCW radar signal is in the form of low-frequency beat tones. The more distant the target, the higher the frequency of the decorrelated beat tone. It is possible to implement a shortduration range gate in a conventional FMCW radar system by placing a band-pass filter (BPF) at the output of the video amplifier, however, this is challenging to implement because it is difficult to design practical high-circuit-Q BPFs at base-band. Higher performance BPFs are available as IF communications filters which operate at high frequencies and are found in two-way radios and various types of communication and television receivers. Examples include crystal, 


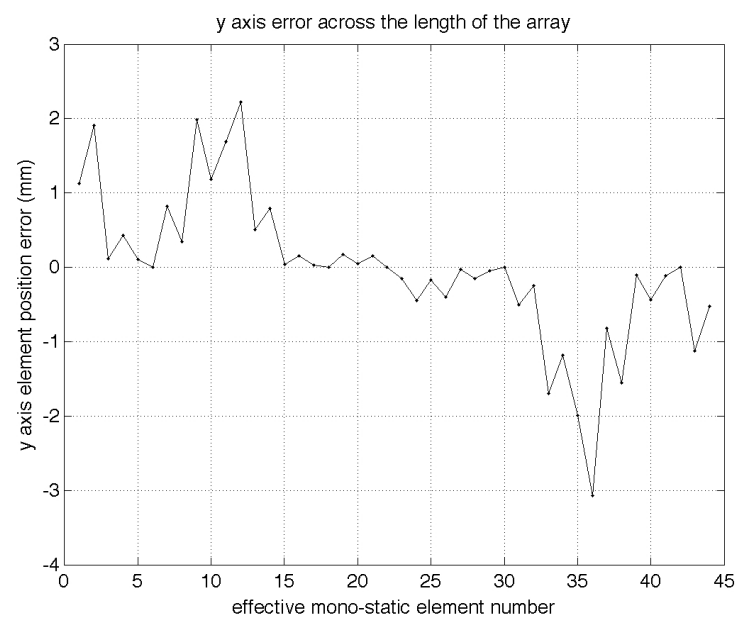

(a)

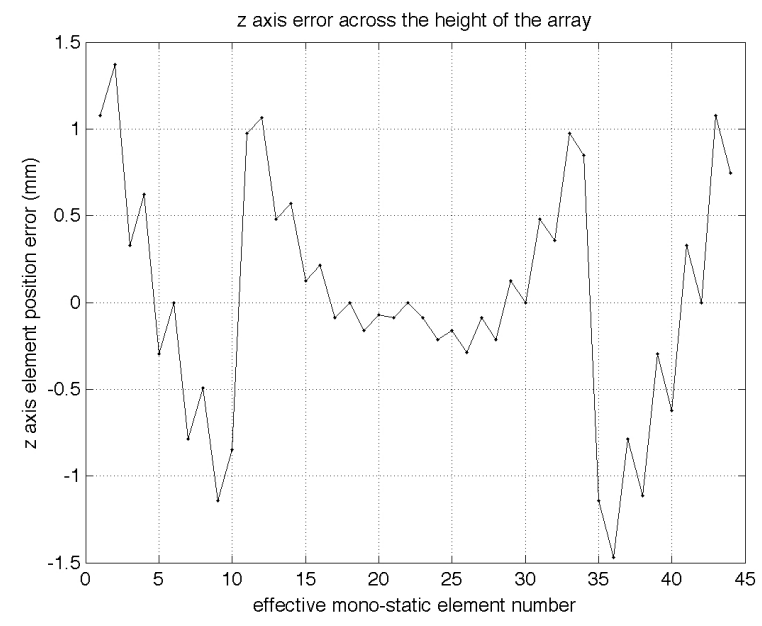

(b)

Fig. 5. Effective mono-static element position error along the y-axis (a) and along the $\mathrm{x}$-axis (b).

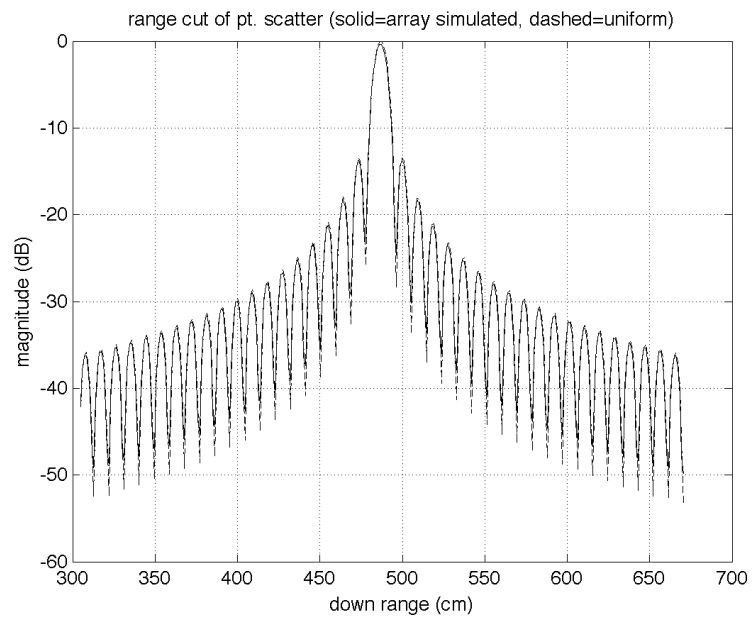

Fig. 6. Down-range cut of a simulated point target.

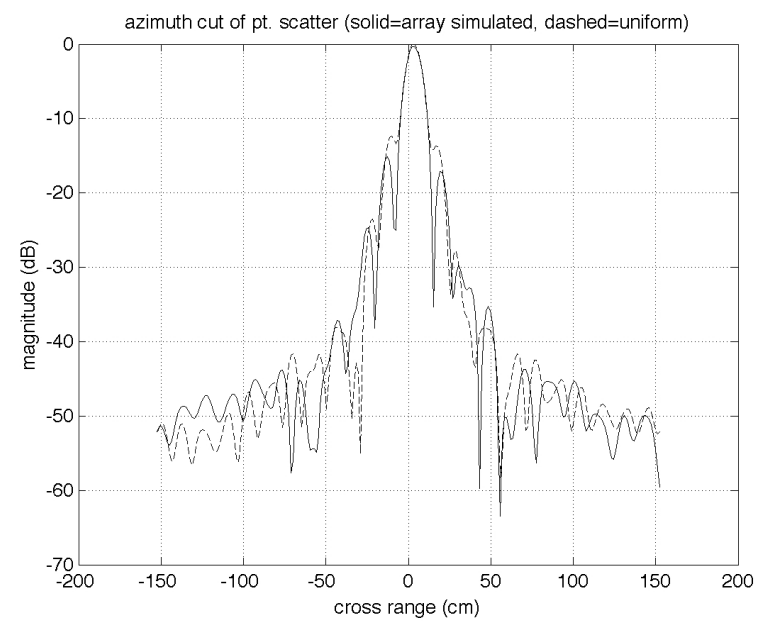

Fig. 7. Azimuth cut of a simulated point target.

ceramic, SAW, and mechanical filters. The radar architecture developed in [1] uses high-Q IF filters as an analog rangegate to reject the reflection off of the air-slab boundary, thereby, providing increased sensitivity and dynamic range for the target scene behind the slab. This narrow IF bandwidth provides a short-duration range gate for the long duration LFM chirp waveforms produced by this radar system. This radar is capable of chirping from $1.926 \mathrm{GHz}$ to $4.069 \mathrm{GHz}$ in $2.5 \mathrm{~ms}$, $5 \mathrm{~ms}$, and $10 \mathrm{~ms}$ providing chirp rates $\left(c_{r}\right)$ of $857 \mathrm{GHz} / \mathrm{s}, 428$ $\mathrm{GHz} / \mathrm{s}$, and $214 \mathrm{GHz} / \mathrm{s}$ respectively. The IF filter has a center frequency $\left(f_{c}=10.7 \mathrm{MHz}\right)$ and a bandwidth $(B W=7.5$ $\mathrm{kHz}$ ) providing a range gate of $8.75 \mathrm{~ns}$ for a chirp rate of 857 $\mathrm{GHz} / \mathrm{s}, 17.5 \mathrm{~ns}$ for a chirp rate of $428 \mathrm{GHz} / \mathrm{s}$, and $35 \mathrm{~ns}$ for a chirp rate of $214 \mathrm{GHz} / \mathrm{s}$. Range gating is represented by the time-domain output of the video amplifier in Fig. 1 (neglecting amplitude terms) by [1]

$$
\begin{aligned}
& \operatorname{Video}(t)= \\
& \begin{cases}\cos \left(2 \pi c_{r} t_{\text {delay }} t\right) & \text { if } \begin{array}{l}
\frac{-B W}{2}+f_{c}-f_{B F O} \\
<c_{r} t_{\text {delay }}<\frac{B W}{2}+f_{c}-f_{B F O} \\
0
\end{array} \\
\text { for all other values, }\end{cases}
\end{aligned}
$$

where $f_{B F O}=10.7 \cdot 10^{6}+c_{r} \cdot t_{\text {rangegate }}$ and $t_{\text {rangegate }}$ is the round-trip time from the front of the radar to the center of the range gate. Furthermore, the use of a long duration LFM chirp allows for a low effective noise bandwidth after pulse compression. Additional gain is achieved by the SAR image processing which effectively compresses received data in the slow-time domain.

The switched-antenna-array radar system is shown in Fig. 8 and the front of the array is shown in Fig. 9. A Labview graphical user interface (GUI) controls the switch matrices, pulses the transmitter, digitizes the de-chirped video signal, computes then displays the SAR image. Calibration and back- 


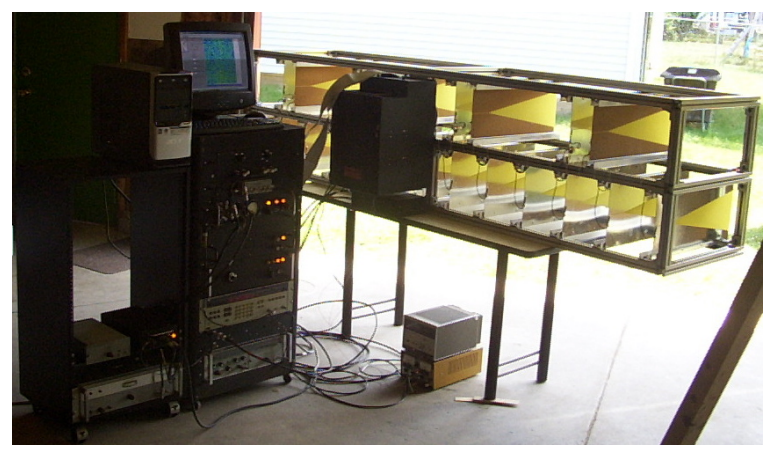

Fig. 8. The real-time S-band radar imaging system.

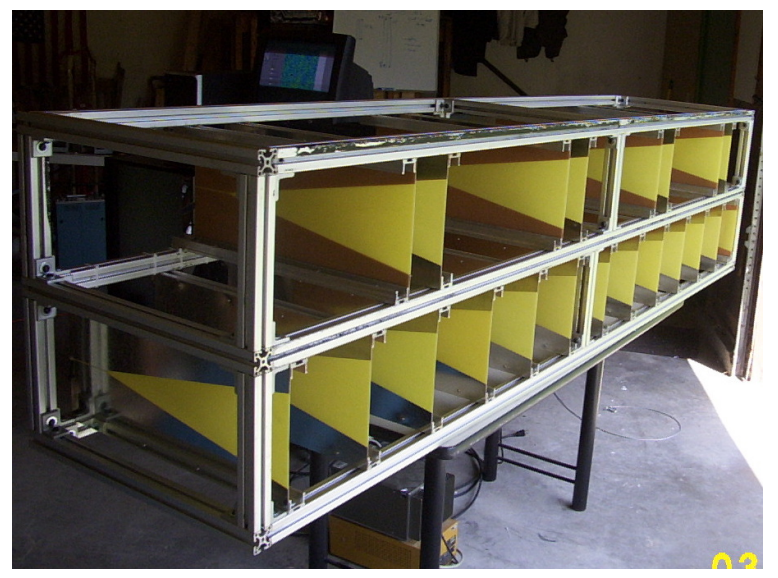

Fig. 9. Front view of the MIMO antenna array.

ground subtraction are applied to each set of 44 range profiles. SAR imagery is displayed on the computer screen shown in Figure 8 . This process is repeated indefinitely in a 'while' loop until the user stops the program. With a $2.5 \mathrm{mS}$ chirp-time this radar system is capable of near-real-time radar imaging at a rate of one image every 1.9 seconds. Complete details of the system implementation are presented in [25].

\section{Calibration}

Calibration compensates for the variation in round-trip delays due to the diverse baselines of the bi-static combinations shown in Fig. 3. One example would be comparing ANT3 and ANT16 to ANT3 and ANT15. The baseline between ANT3 and ANT16 is much longer than ANT3 and ANT15. This causes the round-trip delay to target from ANT3 and ANT16 to be greater than ANT3 and ANT15. Calibration of every bi-static antenna pair to a single point scatterer in a known location compensates for this, allowing the switched array to function like linear array. Calibration is achieved by placing a $1.52 \mathrm{~m}$ tall $1.9 \mathrm{~cm}$ diameter metal pole exactly $3.35 \mathrm{~m}$ down range and centered to the middle of the array. Range profile data is acquired across the array at each of the 44 effective mono-static elements, where it is assumed that the effective mono-static phase centers are evenly spaced every $5.08 \mathrm{~cm}$ and their $\mathrm{y}$-axis positions are represented by $y(n)$. This data is represented by $E_{\text {sPole }}(y(n), \omega(t))$ where $y(n)$ is the crossrange effective mono-static element position. The pole is then removed and a background data set is acquired, represented by $E_{s \text { Calback }}(y(n), \omega(t))$. The background is subtracted from the pole resulting in a $2 \mathrm{D}$ range profile data set of the pole only,

$$
\begin{aligned}
E_{\text {sCal }}(y(n), \omega(t))=E_{\text {sPole }} & (y(n), \omega(t)) \\
& -E_{\text {sCalback }}(y(n), \omega(t)) .
\end{aligned}
$$

The calibration data is referenced to a theoretical point scatterer

$$
E_{\text {sCaltheory }}(y(n), \omega(t))=e^{-j 2 k_{r} R_{\text {pole }}}
$$

where $k_{r}=\omega(t) / c$ and $R_{\text {pole }}$ is a $2 \mathrm{D}$ range to pole across all effective mono-static element positions $y(n)$ represented by the equation

$$
R_{\text {pole }}=\sqrt{y(n)^{2}+(3.35)^{2}} .
$$

The calibration factor is

$$
E_{\text {sCalfactor }}(y(n), \omega(t))=\frac{E_{\text {sCaltheory }}(y(n), \omega(t))}{E_{\text {sCal }}(y(n), \omega(t))} .
$$

This coefficient is applied to the SAR data acquired from the array by multiplying it by each set of 44 range profiles acquired.

\section{RESUlTS}

Simulations are in agreement with measured imagery for close-in down-range and cross-range sidelobes. In addition, imaging scenes of targets as small as $3.18 \mathrm{~cm}$ tall nails is possible in free space.

\section{A. Sidelobes}

A measured free-space image of a soda can located at 484.2 $\mathrm{cm}$ down range and $2.5 \mathrm{~cm}$ cross range is shown in Fig. 10b. For the analysis presented here it is assumed that this is a point target because of the range resolution, operational frequency, and length of this phased array radar. The simulated image of a point scatterer using the methods from Section II is shown in Fig. 10a.

Measured and simulated down-range responses are shown in Fig. 11. The measured location of the soda can is slightly shifted towards the array. This could be due to errors in the expected physical down range location of the calibration pole. Some increases in range sidelobe levels are indicated at 540, 600,650 , and $675 \mathrm{~cm}$. These are likely due to clutter in the target scene (measurements were acquired outdoors in the author's backyard). From this it is clear that the measured down-range resolution is in agreement with simulation.

Measured and simulated cross-range responses are shown in Fig. 12. The further out sidelobes starting around $\pm 50 \mathrm{~cm}$ do not agree with simulation. This could be due to mutual coupling between elements, feed lines, and antenna switches. The switches used in this system are designed to work up to $3 \mathrm{GHz}$ with $40 \mathrm{~dB}$ of port-to-port isolation. It was decided to push the limit of operation of these switches to $4 \mathrm{GHz}$ because 


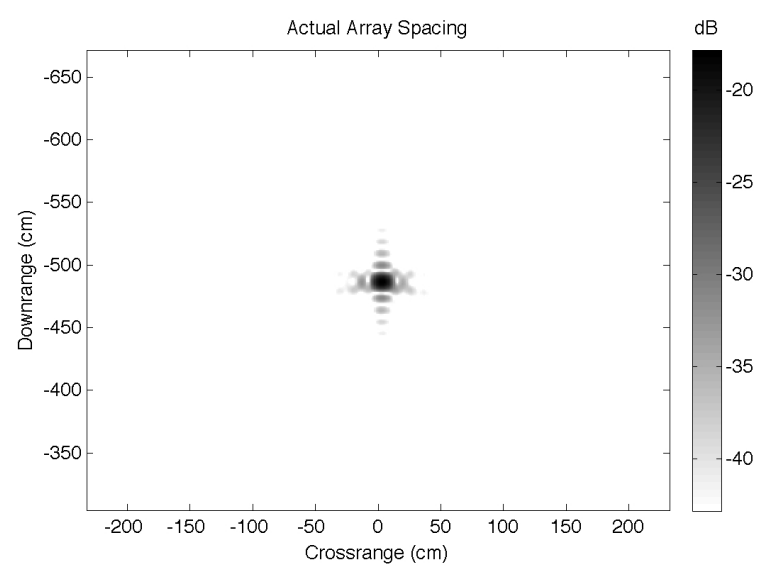

(a)

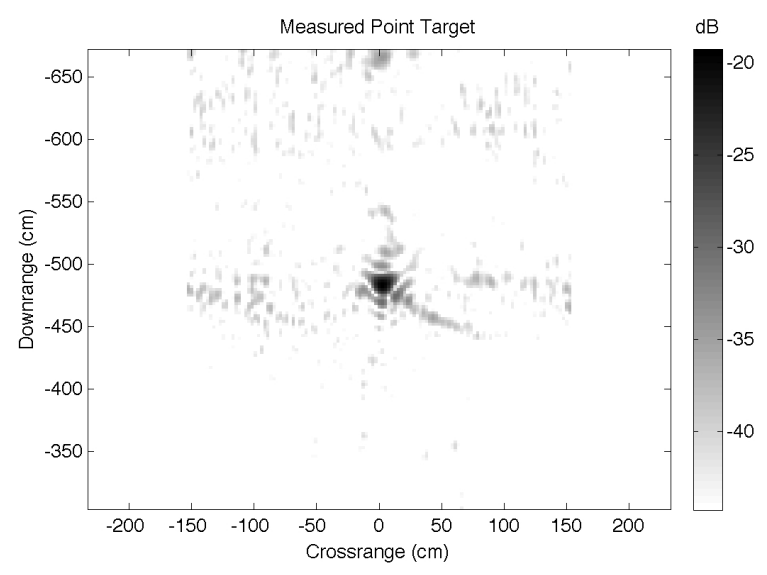

(b)

Fig. 10. A near-real-time SAR image of a point target: simulated (a), measured (b).

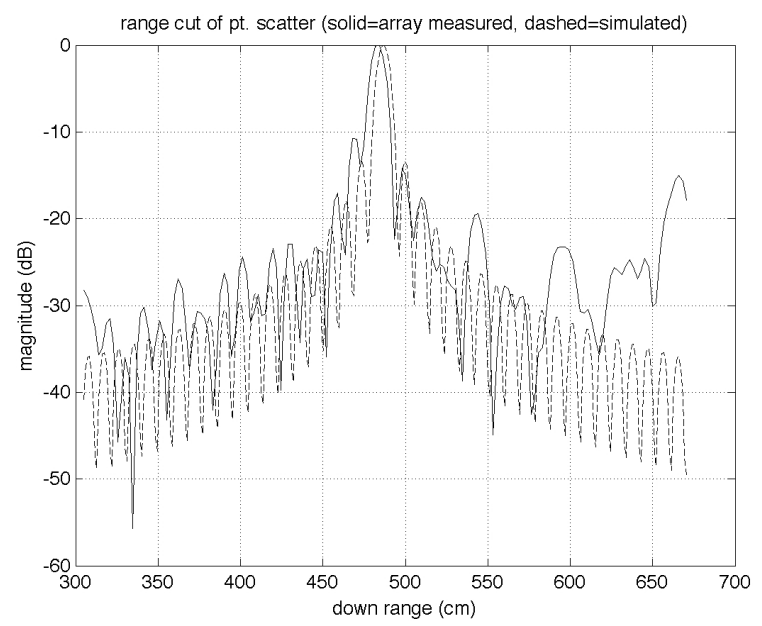

Fig. 11. Simulated and measured down range cuts of a point target.

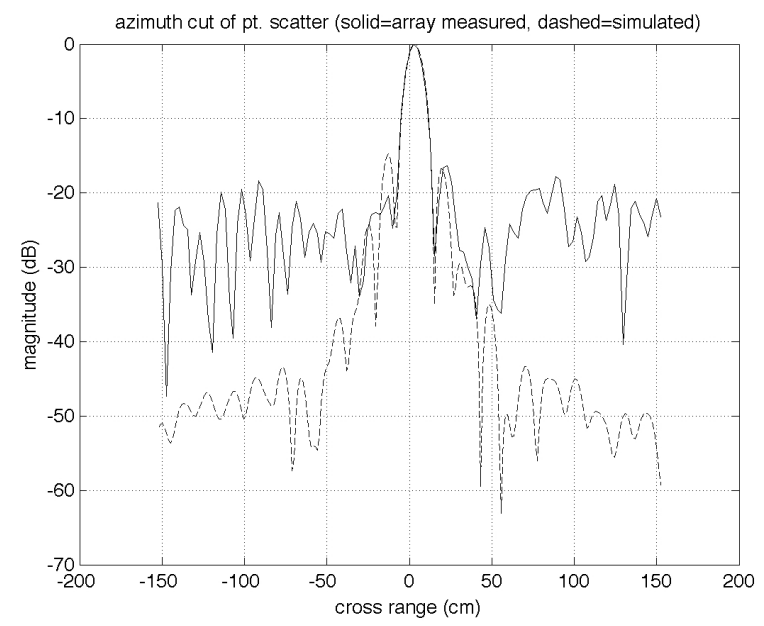

Fig. 12. Simulated and measured cross range cuts of a point target.

of funding and time restrictions. At $4 \mathrm{GHz}$ the port-to-port isolation drops to about $35 \mathrm{~dB}$, increasing mutual coupling due to the switches. Further coupling could be due to the fact that the element feed lines are bundled together on one wiring harness which is fed across the middle support member on the rear of the array. Regardless of this coupling, the closein sidelobes and the $-3 \mathrm{~dB}$ points agree with simulation; therefore the measured cross-range resolution is in agreement with simulation.

\section{B. Resolution}

In this section, a free-space image of a block-S configuration of 14 carriage bolts with equal spacing of approximately 0.305 $\mathrm{m}$ between adjacent bolts are discussed to characterize the radar's resolution. The bolts are mounted on a styrofoam board $\left(\epsilon_{r}=1.03[26]\right)$ that is parallel to the ground and approximately $5.25 \mathrm{~m}$ from the antenna array. Furthermore, the board is essentially electromagnetically transparent at the radar transmission frequencies (1.926-4.069 GHz). Each bolt is $15.24 \mathrm{~cm}$ long with a diameter of $0.95 \mathrm{~cm}$. Each bolt is mounted vertically providing a small down range and cross range extent allowing the bolts to act like point scatterers. Imaging these bolts allows the radar's resolution to be tested.

The range resolution was measured by irradiating the target scene with $1 \mathrm{~mW}$ of transmit power resulting in the image shown in Fig. 13. The expected range resolution depends on the chirp bandwidth calculated using the equation [22]

$$
\rho_{r}=\frac{c}{2 B},
$$

where $B$ is the chirp bandwidth $(=2.143 \mathrm{GHz})$. The expected down range resolution based on chirp bandwidth is $7 \mathrm{~cm}$. The down range resolution measured from this image is $9.8 \mathrm{~cm}$. These results show that the switched-antenna-array radar is performing close to the smallest theoretical range resolution possible.

The cross range resolution depends on the length of the array $L(=2.24 \mathrm{~m})$ and the distance of the point target to 


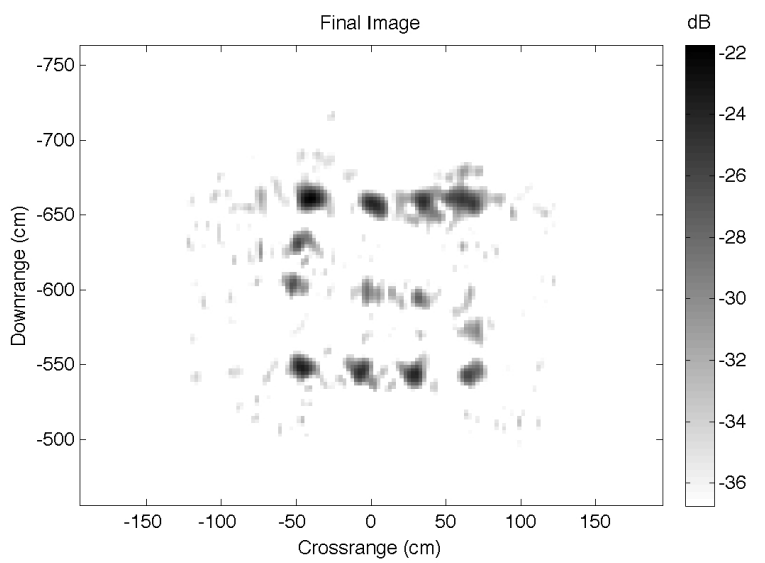

Fig. 13. SAR image of a group of $15.24 \mathrm{~cm}$ long $0.95 \mathrm{~cm}$ diameter carriage bolts in free-space.

the front of the array in both down range and cross range. A SAR imaging algorithm is used for beamforming, from this cross-range resolution is calculated by [22]

$$
\rho_{c r}=\frac{\lambda R_{t}}{2 L \sin \phi_{d c} \cos (\Delta \theta / 2)},
$$

where $R_{t}$ is the range to point target, $\phi_{d c}$ is the angle from the center of the aperture to the point target, and $\Delta \theta$ is the change in target aspect angle from 0 to $L$ across the aperture. The expected cross-range resolution for all targets shown at $546 \mathrm{~cm}$ is $12.5 \mathrm{~cm}$. The measured cross range is $10.2 \mathrm{~cm}$. The expected cross-range resolution for all targets shown at $661 \mathrm{~cm}$ down range is $15 \mathrm{~cm}$. The measured cross-range is $12 \mathrm{~cm}$. These results show that this switched-antenna-array radar is performing close to the smallest theoretical crossrange resolution possible.

\section{Low RCS imagery}

Additional free-space SAR imagery was acquired to test the radar's sensitivity for target scenes with multiple low RCS point targets. Figure 14 shows an image of a target scene made up of $5.08 \mathrm{~cm}$ tall metal nails in the same block ' $\mathrm{S}$ ' configuration. The position of each nail is clearly shown, where the last two rows are fading into the noise and clutter.

Figure 15 shows a SAR image of a group of $3.18 \mathrm{~cm}$ tall nails in a block ' $\mathrm{S}$ ' configuration. The bottom two rows are clearly shown and a few of the nails at the top row are noticeable. The fact that these images could be acquired using $1 \mathrm{~mW}$ of peak transmit power demonstrates the radar's sensitivity.

\section{CONCLUSiOnS}

A though-slab rail SAR imaging system was developed in [1] using a modified FMCW radar architecture which facilitated through-slab imaging at stand-off ranges however; this system was too slow for practical applications. In this paper a

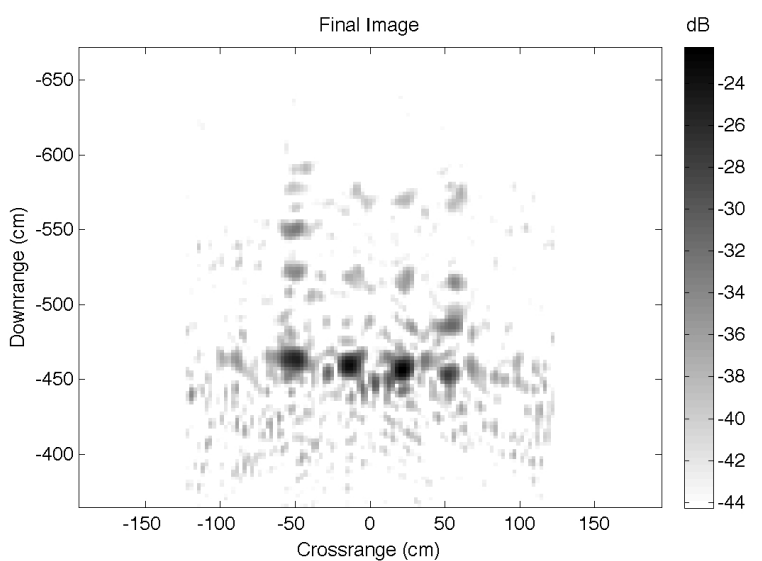

Fig. 14. Image of a group of $5.08 \mathrm{~cm}$ tall nails in free-space.

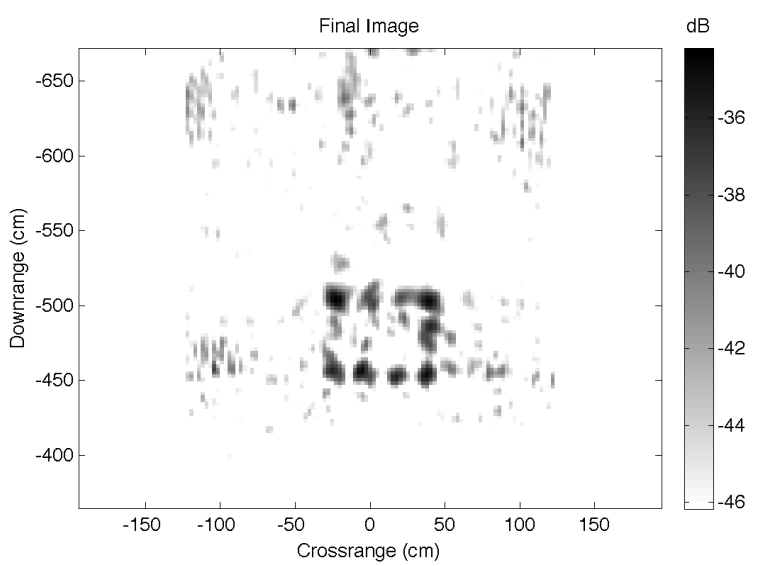

Fig. 15. Image of a group of $3.2 \mathrm{~cm}$ tall nails in free-space.

near-real time switched-antenna phased array radar system was developed using this FMCW radar which provides near-realtime imaging, reducing the data acquisition time from 20 minutes to less than 2 seconds. This was achieved by multiplexing the transmit and receive ports of the FMCW radar across a multitude of discrete antenna elements. Simulations of point scatterers in free-space showed negligible difference between an ideal linear array and the switched array. Measurements of a point target agreed with simulations with respect to range resolution and down-range side lobes, but with increased farout cross-range sidelobes likely due to mutual coupling (both in the RF signal chain and on the array). In addition to this, low RCS target scenes in free-space were imaged using only $1 \mathrm{~mW}$ of peak transmit power demonstrating sensitivity. Future work will include applying the through-slab model from [1] to the switched antenna array to characterize expected performance in a through-slab scenario. Additional future work will include increasing the imaging re-fresh rate to greater than five images 
per second by upgrading to higher throughput data acquisition hardware.

\section{ACKNOWLEDGMENT}

The authors would like to thank the Naval Research Laboratory with funding from the Office of Naval Research, ONR Code 30, the Expeditionary Maneuver Warfare \& Combating Terrorism Department, for funding this project.

\section{REFERENCES}

[1] G. L. Charvat, L. C. Kempel, E. J. Rothwell, C. Coleman, and E. L. Mokole, "A through-dielectric radar imaging system," IEEE Transactions on Antennas and Propagation, Accepted January 31, 2010.

[2] P. J. F. Swart, J. Schier, A. J. van Gemund, W. F. van der Zwan, J. P. Karelse, G. L. Reijns, P. van Genderen, L. P. Ligthart, and H. T. Steenstra, "The Colorado multistatic FMCW radar system," IEEE European Microwave Conference, October 1998, Vol. 2, pp. 449-454.

[3] V. Katkovnik, M. S. Lee, and Y. H. Kim, "High-resolution signal processing for a switch antenna array FMCW radar with a single channel receiver," IEEE Proceedings of Sensor Array and Multichannel Signal Processing, 4-6 August 2002, pp. 543-547.

[4] M. S. Lee, V. Katkovnik, and Y. H. Kim, "System modeling and signal processing for a switch antenna array," IEEE Transactions on Signal Processing, Vol. 52, Issue 6, June 2004, pp. 1513-1523.

[5] M. A. Barnes, S. Nag, and T. Payment, "Covert situational awareness with handheld ultra-wideband short pulse radar," Radar Sensor Technology VI, Proceedings of SPIE Volume 4374, 2001.

[6] S. Nag, M. A. Barnes, T. Payment, and G. W. Holladay, "An ultrawideband through-wall radar for detecting the motion of people in real time," Radar Sensor Technology and Data Visualization, Proceedings of SPIE Volume 4744, 2002.

[7] A. R. Hunt, "Image formation through walls using a distributed radar sensor array," Proceedings of the 32nd IEEE Applied Imagery Pattern Recognition Workshop, 15-17 October 2003, pp. 232-237.

[8] M. Mahfouz, A. Fathy, Y. Yang, E. E. Ali, and A. Badawi, "See-throughwall imaging using ultra wideband pulse systems," IEEE Proceedings of the 34th Applied imagery and Pattern Recognition Workshop, 2005.

[9] R. Benjamin, I. J. Craddock, E. McCutcheon, and R. Nilavalan, "Throughwall imaging using real-aperture radar," Sensors, and Command, Control, Communications, and Intelligence (C3I) Technologies for Homeland Security and Homeland Defense IV, Proceedings of SPIE Volume 5778, 20 May 2005.

[10] Y. Yang, C. Zhang, S. Lin, and A. E. Fathy "Developement of an ultra wideband Vivaldi antenna array," IEEE Antennas and Propagation Society International Symposium, Vol. 1A, 2-8 July 2005, pp. 606-609.

[11] A. Berri and R. Daisy, "High-resolution through-wall imaging," Sensors, and Command, Control, Communications, and Intelligence (C3I) Technologies for Homeland Security and Homeland Defense V, Proceedings of SPIE Volume 6201, 6201J, 2006.

[12] H. Burchett, "Advances in through wall radar for search, rescue and security applications," The Institution of Engineering and Technology Conference on Crime and Security, 13-14 June 2006, pp. 511-525.

[13] W. Zhiguo, L. Xi, and F. Yuanchun, "Moving target position with through-wall radar,' Proceedings of the CIE '06 International Conference on Radar, Oct. 2006.

[14] Y. Yang and A. Fathy, "Design and implementation of a lowcost real-time ultra-wide band see-through-wall imaging radar system," IEEE/MTT-S International Microwave Symposium, 3-8 June, 2007, pp.1476-1470.

[15] R. Janaswamy, D. H. Schaubert, and D. M. Pozar, "Analysis of the transverse electromagnetic mode linearly tapered slot antenna," Radio Science, Volume 21, No. 5, pp. 797-804, September-October 1986.

[16] R. Janaswamy and D. H. Schaubert, "Analysis of the tapered slot antenna," IEEE Transactions on Antennas and Propagation, Vol. AP-35, No. 9, September 1987, pp. 1058-1065.

[17] R. Janaswamy, "An accurate moment method model for the tapered slot antenna," IEEE Transactions on Antennas and Propagation, Vol. 37, No. 12, December 1989, pp. 1523-1528

[18] Y. S. Kim and S. Yngvesson, "Characterization of tapered slot antenna feeds and arrays," IEEE Transactions on Antennas and Propagation, Vol. 38, No. 10, October 1990, pp. 1559-1564.
[19] P. S. Kooi, T. S. Yeo, and M. S. Leong, "Parametric studies of the linearly tapered slot antenna (LTSA)," IEEE Microwave and Optical Technology Letters, Vol. 4, No. 5, April 1991, pp. 200-207.

[20] X. D. Wu and K. Chang, "Compact wideband integrated active slot antenna amplifier," IEEE Electronics Letters, Vol. 29, No. 5, 4 March 1993, pp. 496-497.

[21] E. Thiele and A. Taflove, "FD-TD analysis of Vivaldi flared horn antennas and arrays," IEEE Transactions on Antennas and Propagation, Vol. 1, Nov. 2003, pp. 54-59.

[22] W. G. Carrara, R. S. Goodman, and R. M. Majewski, Spotlight Synthetic Aperture Radar Signal Processing Algorithms, Artech House, Boston, MA, 1995.

[23] N. J. Willis, Bistatic Radar, Scitech Publishing, Inc., Raleigh, NC, 1995.

[24] E. W. Weisstein, "Angle bisector theorem." From MathWorld-A Wolfram Web Resource. http://mathworld.wolfram.com/AngleBisectorTheorem.html.

[25] G. L. Charvat, "A Low-Power Radar Imaging System," Ph.D. dissertation, Deptartment of Electrical and Computer Engineering, Michigan State University, East Lansing, MI, 2007.

[26] C.A. Balanis, Advanced Engineering Electromagnetics, John Wiley \& Sons, New York, NY, 1989. 